\title{
Mechanical forces exerted by a dipole emitter on an interface
}

Dmitri Petrov

Dmitri.Petrov@icfo.es

\author{
ICFO - Institut de Ciencies Fotoniques, Mediterranean Technology Park, 08860, \\ Castelldefels (Barcelona), Spain \\ ICREA - Institució Catalana de Recerca i Estudis Avançats, 08010, Barcelona, Spain
}

Mechanical forces exerted by an emitting dipole on the interface between two media with different dielectric susceptibilities are analyzed for different distances between the dipole and the interface. Estimations of the force values are given based on known molecular polarizabilities for inelastic processes such as Raman scattering and fluorescence including those that occur near metal structures. [DOI: 10.2971/jeos.2010.10028]

Keywords: radiation force, Maxwell stress tensor, dipole field

The conventional way to characterize fluorescence and Raman processes is through the analysis of a spectrum of light reflected from a scatterer. However, one can consider a different physical perspective where the fluorescence and Raman scattering result in a change of the total momentum of the scattering field due to the emission of the photons. Any changes of the total scattering field by a given incident field induces mechanical forces that are exerted on the scatterer [1]. Hence, the inelastic processes contain a momentum exchange between the incident and scattered photons and the scatterer; therefore a measure of the radiation forces acting on the scatterer is equivalent to the measurement of its recoil that is exerted by the emitted photons.

Recent experiments [2,3] demonstrated that the sensitivity of existing techniques is sufficient to detect mechanical effects related with a transfer of momentum by emission of Raman or fluorescence photons. A photonic force microscope $[4,5]$ coupled with a spectroscopic system measured simultaneously the radiation forces exerted on, and the emission from a micron sized scatterer (a dielectric bead partially covered by metal colloids) optically trapped by a focused beam in a liquid with dye molecules. The metal nanosized colloids increase the efficiency of the inelastic processes due to surface plasmon resonances as well as to changes of molecular polarizabilities caused by the adsorption of the molecules onto the metal surface [6].

However, physical mechanisms involved in the momentum transfer from emitting molecules to a scatterer of the photonic force microscope and the sensitivity of the technique have not been discussed yet in details. Here we propose a simple model that may be as the starting point for more exact calculations. It is well known that phenomenological theories of Raman and fluorescence emission explain radiation processes starting from the radiation emitted by an oscillating dipole [6, 7]. The dipole is highly localized and its moment is defined by local excitation fields and local values of the molecular polarizabilities for a given emission process. The model proposed does not include details of all physical effects accompanied the experiments, but it captures the key element - the radiation pressure of a dipole on an interface. A didactic model of the radiation mechanical effect - the radiation pressure of a plane wave on a flat surface - can not describe, for example, the gradient force produced by the dipole, as we show in this work. Any other more developed approach may be included in our model by changing the polarizability of the emitting dipole and its orientation. Although we consider that the surface that accepts the dipole radiation pressure as an infinite and flat one, the pressure on the surface of limited sizes may be calculated.

In the experiments $[2,3]$ the emission sources are localized near those parts of metal structures at the surface of the scatterer where the maximal enhancement of the incident and emitted radiations occurs (see Figure 1(a)). We assume that the recoil effect observed experimentally is due to the radiation force exerted by the localized dipole emitters on the surface of the scatterer. The goal of the present study is to estimate this radiation force.

The proposed model, obviously, is too simple in order to be used by direct comparison with known experimental results. The surface emission enhancement are generally related to more complex geometries, such as strong curvatures or two nearby interfaces, as shown in Figure 1(a). However, although this enhancement can be only near curvatured surfaces, we consider that the emitted field exerts the pressure at the flat surface due to the following reasons. In experiments $[2,3]$ the irregular metal strusctures with characteristic sizes of several tens of nanometers are at the surface of the dielectic bead of $2 \mu \mathrm{m}$ in diameter. Keeping in mind this difference in the sizes of the metal structures and the interface where these nanostructures are located one can treat a surface that accepts the radiation pressure of the emitted fields as a flat one. It also permits one to neglect the radiation pressure on the metal irregular structures because their total surface is much smaller that the surface of the sphere. 


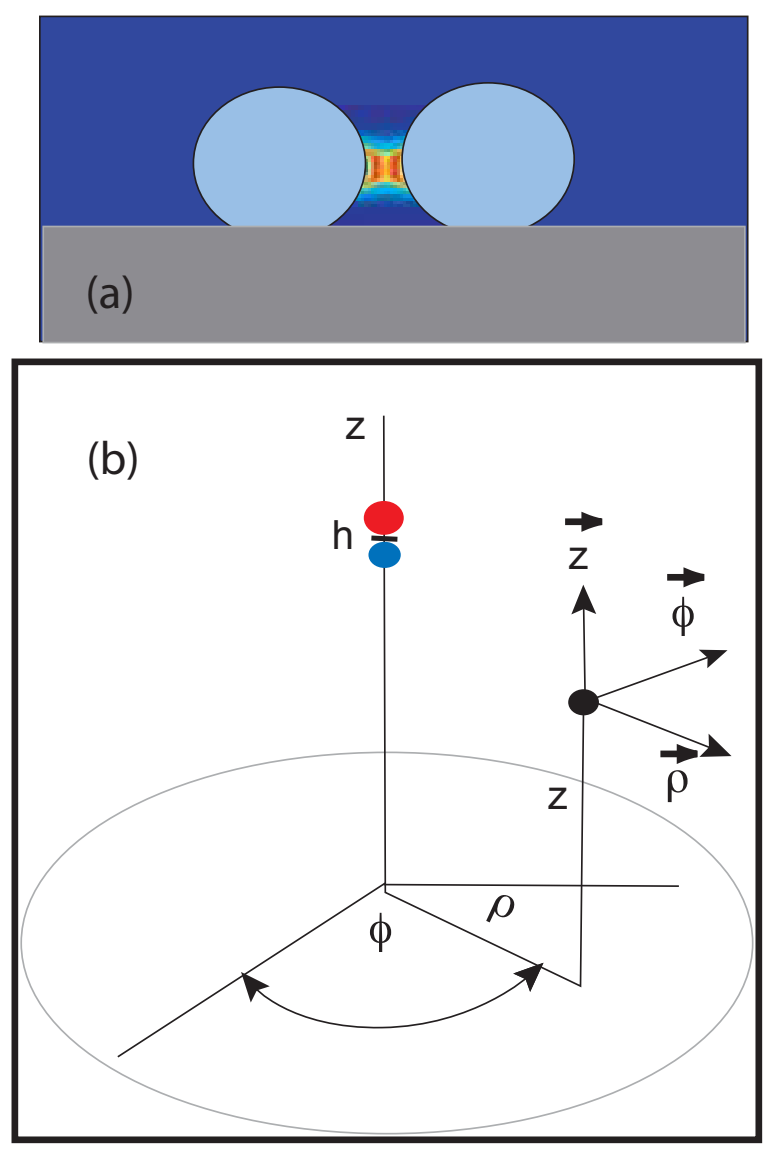

FIC. 1 (a) Strong fields between two metal colloids where an emitting molecule is located; (b) A geometry of the problem: $(\phi, \rho, z)$ are the cylindrical coordinates of the observation point $z$ and the dipole with vertical moment is at $(0,0, h)$.

The interaction of a dipole with interfaces has been very well studied in relation with various aspects, for example, the nearfield spectroscopy [8] or lifetime of an emitting dipole near a surface [9], but mechanical effects have not been discussed yet. In the following we start with known expressions for the fields of a dipole near the interface between two media with different dielectric susceptibilities [10] in order to find the Maxwell stress tensor. Then by integrating the Maxwell tensor component normal to the interface over the whole interface, we find the radiation force exerted on the surface. Finally, using known values of molecular polarizability we estimate the mechanical force including also the cases when the inelastic processes occur near metal structures. The model proposed here neglects the momentum recoil of the emitting dipole itself $[11,12]$ considering the dipole just as a source of electromagnetic field located at aiven distance from the interface.

We suppose that a vertical dipole with (induced) dipole moment $p_{0}$ is located in a medium $(z>0)$ with relative dielectric susceptibility $\varepsilon_{1}=n_{1}^{2}$ (without losses $\operatorname{Im}\left[n_{1}\right]=0$ ) at a distance $h$ from the interface with a medium $(z<0)$ with relative dielectric susceptibility $\varepsilon_{2}=N^{2}$, where $\varepsilon_{0}$ is the vacuum dielectric susceptibility, and $N$ is the complex refractive index (see Figure 1(b)). The choice of the vertical polarization simplifies calculations, but in the experiments the orientation of the dipole includes both the vertical and horizontal components and is dictated by the specific of the electromagnetic field in the gap between the metal structures and by the polarization of the incident field. Nevertheless the model with this polar- ization captures the main crucial difference from the didactic example of the radiation pressure of a plane wave on a plane interface: the presence both the gradient force and the scattering force exert on the interface due to the specific properties of the dipole emission.

The dipole moment $p_{0}=\alpha E_{\text {loc }}$, where $\alpha$ is the molecular polarizability for the Raman scattering (or fluorescence) and $E_{\mathrm{loc}}$ is the local electrical field that excites the dipole and its value is defined by the intensity of the incident field and may be modified by the enhancement of the field due to the plasmonic effects in the metal structures. The value of $\alpha$ may also affected by the interaction of the molecule with the metal and also depends on the number of the molecules in this local hot spot. We chose the cylindrical coordinate system with the coordinates $(\rho, \phi)$ in the plane of the interface and the dipole is located at the point with coordinates $(0,0, h)$. The force that the dipole exerts on the interface is given by [10]: $F_{z}=\int_{S} d S\left\langle T_{z z} \cdot n_{z}\right\rangle$ where the interface $S$ is supposed to be infinite in extend and thus accounts for the total momentum of the dipole field flowing through it. Here $T$ is the Maxwell stress tensor

$$
\begin{aligned}
\left\langle T_{\alpha \beta}\right\rangle=\frac{1}{2} \varepsilon_{1} \varepsilon_{0} \operatorname{Re}\left[E_{\alpha}^{*} E_{\beta}\right. & +\frac{1}{\mu_{0} \varepsilon_{1} \varepsilon_{0}} B_{\alpha}^{*} B_{\beta} \\
& \left.-\frac{1}{2}\left(\mathbf{E}^{*} \cdot \mathbf{E}+\frac{1}{\mu_{0} \varepsilon_{1} \varepsilon_{0}} \mathbf{B}^{*} \cdot \mathbf{B}\right) \delta_{\alpha \beta}\right],
\end{aligned}
$$

and \langle\rangle denotes the time averaging. $\mathbf{E}$ and $\mathbf{B}$ are the electric and magnetic fields of the dipole. The fields are expressed through the Hertz vector $\Pi$ that for the vertical dipole has only $z$-component. We need the field at the interface $(z=0)$ therefore we use the Hertz vector for the space between $z=0$ and $z=h$, that is given by [10]

$$
\Pi_{z}=\frac{p_{0}}{4 \pi \varepsilon_{0} \varepsilon_{1}} \int_{0}^{\infty} d k_{\rho} J_{0}\left(k_{\rho} \rho\right) X\left(k_{\rho}\right)
$$

with

$$
X\left(k_{\rho}\right)=i \frac{k_{\rho}}{k_{z}}\left[\exp \left(-i k_{z} z\right)+r^{(p)} \exp \left(i k_{z} z\right)\right] \exp \left(i k_{z} h\right),
$$

where $k_{z}=\left(k_{0}^{2} n_{1}^{2}-k_{\rho}^{2}\right)^{1 / 2} \cdot k_{0}=\omega / c$ is the wavenumber in vacuum and $\operatorname{Im}\left[k_{z}\right]>0$ to provide the convergence of the fields, $r^{p}$ is the Fresnel reflection coefficient for p-polarized plane waves [10] $r^{(p)}=\left(a\left(k_{\rho}\right)-b\left(k_{\rho}\right)\right) /\left(a\left(k_{\rho}\right)+b\left(k_{\rho}\right)\right)$ with

$$
a\left(k_{\rho}\right)=\sqrt{\left(\operatorname{Re}[N]^{2}+\operatorname{Im}[N]^{2}\right) k_{0}^{2}-k_{\rho}^{2}+i \frac{2 \operatorname{Re}[N] \operatorname{Im}[N] k_{\rho}^{2}}{\operatorname{Re}[N]^{2}+\operatorname{Im}[N]^{2}}}
$$

and

$$
b\left(k_{\rho}\right)=\frac{\sqrt{\operatorname{Re}[N]^{2}+\operatorname{Im}[N]^{2}}(\operatorname{Re}[N]+i \operatorname{Im}[N])}{n_{1}^{2}} \sqrt{k_{0}^{2} n_{1}^{2}-k_{\rho}^{2}} .
$$

The Hertz vector includes both propagating fields (when $0<$ $k_{\rho}<k_{1}$ ) and evanescent fields (when $k_{1}<k_{\rho}$ ).

There are three nonzero components of the electric $E$ and mag- 
netic $H$ fields

$$
\begin{aligned}
& H_{\phi}(z, \rho)=i \omega \frac{p_{0}}{4 \pi} \int_{0}^{\infty} d k_{\rho} k_{\rho} J_{1}\left(k_{\rho} \rho\right) X\left(k_{\rho}, z\right), \\
& E_{z}(z, \rho)=\frac{p_{0}}{4 \pi \varepsilon_{0} \varepsilon_{1}} \int_{0}^{\infty} d k_{\rho} J_{0}\left(k_{\rho} \rho\right) \frac{\partial^{2}}{\partial z^{2}} X\left(k_{\rho}, z\right), \\
& E_{\rho}(z, \rho)=-\frac{p_{0}}{4 \pi \varepsilon_{0} \varepsilon_{1}} \int_{0}^{\infty} d k_{\rho} k_{\rho} J_{1}\left(k_{\rho} \rho\right) \frac{\partial}{\partial z} X\left(k_{\rho}, z\right) .
\end{aligned}
$$

Using these fields in the Maxwell tensor at $z=0$ and integrating it over the whole interface we found the radiation force exerted on the interface

$$
F_{z}=F^{z z}+F^{\rho \rho}+F^{\phi}
$$

where

$$
\begin{aligned}
F^{z z}= & \frac{1}{4} \varepsilon_{0} \varepsilon_{1} \operatorname{Re}\left[\int_{0}^{\infty} d \rho \rho \int_{0}^{2 \pi} d \phi E_{z}(z, \rho) E_{z}^{*}(z, \rho)\right] \\
= & \frac{p_{0}^{2}}{32 \pi \varepsilon_{0} \varepsilon_{1}} \operatorname{Re}\left[\int_{0}^{k_{1}} d k_{\rho}\left|k_{z}\right|^{2} k_{\rho}\left\{1+r^{(p)}+r^{(p) *}+\left|r^{p}\right|^{2}\right\}\right. \\
& \left.+\int_{k_{1}}^{\infty} d k_{\rho}|\gamma|^{2} k_{\rho}\left\{1+r^{(p)}+r^{(p) *}+\left|r^{p}\right|^{2}\right\} \exp (-2 \gamma h)\right]
\end{aligned}
$$

$$
\begin{aligned}
F^{\rho \rho}= & -\frac{1}{4} \varepsilon_{0} \varepsilon_{1} \operatorname{Re}\left[\int_{0}^{\infty} d \rho \rho \int_{0}^{2 \pi} d \phi E_{\rho}(z, \rho) E_{\rho}^{*}(z, \rho)\right] \\
= & -\frac{p_{0}^{2}}{32 \pi \varepsilon_{0} \varepsilon_{1}} \operatorname{Re}\left[\int_{0}^{k_{1}} d k_{\rho} k_{\rho}^{3}\left\{1-r^{p}-r^{(p) *}+\left|r^{(p)}\right|^{2}\right\}\right. \\
& \left.+\int_{k_{1}}^{\infty} d k_{\rho} k_{\rho}^{3}\left\{1-r^{p}-r^{(p) *}+\left|r^{(p)}\right|^{2}\right\} \exp (-2 \gamma h)\right] \\
F^{\phi}= & \frac{1}{4} \mu_{0} \operatorname{Re}\left[\int_{0}^{\infty} d \rho \rho \int_{0}^{2 \pi} d \phi H_{\phi}(z, \rho) H_{\phi}^{*}(z, \rho)\right] \\
= & -\frac{p_{0}^{2} k_{0}^{2}}{32 \pi \varepsilon_{0}} \operatorname{Re}\left[\int_{0}^{k_{1}} d k_{\rho} \frac{k_{\rho}^{3}}{\left|k_{z}\right|^{2}}\left\{1+r^{(p)}+r^{(p) *}+\left|r^{p}\right|^{2}\right\}\right. \\
& \left.+\int_{k_{1}}^{\infty} d k_{\rho} \frac{k_{\rho}^{3}}{\left|k_{z}\right|^{2}}\left\{1+r^{(p)}+r^{(p) *}+\left|r^{p}\right|^{2}\right\} \exp (2 \gamma h)\right]
\end{aligned}
$$

with $k_{1}=k_{0} n_{1}, \gamma=\sqrt{k_{\rho}^{2}-k_{1}^{2}}$.

Below we illustrate our calculations showing the dependence of the partial contributions $F^{z z}, F^{\rho \rho}, F^{\phi}$, and the total radiation force $F_{z}$ versus the distance between the dipole and the interface between a dielectric medium $(N=1.55)$ and vacuum, and between the gold $(N=-54+5.9 i)$ and vacuum at the wavelength of $0.63 \mathrm{~nm}$. In order to estimate the absolute value of the force one have to know the induced dipole moment $p_{0}$. If we suppose that the incident intensity is equal to $1 \mathrm{~mW}$ and a Gaussian beam is focussed to $1 \mu \mathrm{m}$ in diameter (as in the experiments [2]) the incident local field is equal to $E_{\mathrm{loc}}=10^{6} \mathrm{~V} / \mathrm{m}$. We suppose that a molecule has the induced polarizability around $5 \mathrm{~A}^{3}$ (or $5 \times 10^{-40} \mathrm{Cm}^{2} / \mathrm{V}$ ) [7], then the dipole moment is $p_{0}=5 \times 10^{-34} \mathrm{Cm}$.

The calculations showed that the main contribution to the total force is due to the tangential $E_{\rho}$ component of the dipole field both for the dielectric and metal interface (see Figure 2). The force $F^{\rho \rho}$ is negative which corresponds to the repulsive interaction between the dipole and the interface. The force components $F^{\phi}$ and $F^{z z}$ are much smaller, except the force $F^{z z}$

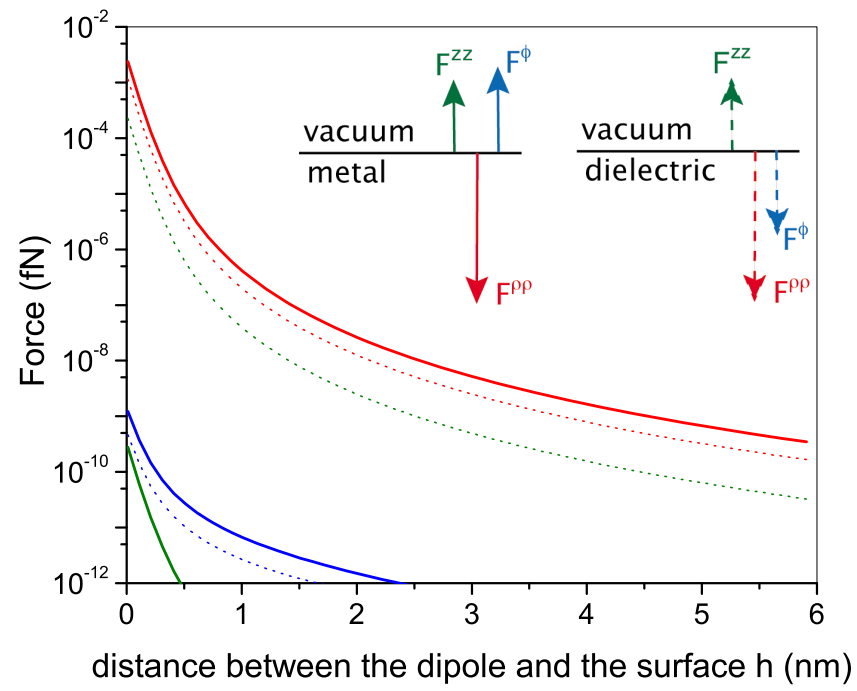

FIG. 2 Contributions of different components of the electromagnetic field of the dipole to the radiation force: $F^{\rho \rho}$ (red lines), $F^{\phi}$ (blue lines) and $F^{z z}$ (green lines) when the medium at $z<0$ is the metal (solid lines) or the dielectric (dot lines). The inset shows the directions of the different contributions to the total force for the interface with the metal (solid lines) and with the dielectric (dashed lines).

for the dielectric interface which is negative. The component $F^{\phi}$ caused by the tangential component of the dipole field $E_{\phi}$ is positive both for the metal and dielectric interfaces, as well as the $F^{z z}$ for the metal interface. Notice, that the forces decreases several orders of magnitude when the dipole is moved from the interface at the distance of $2-3 \mathrm{~nm}$. The components of the force with positive sign are very small compared with the components with negative sign, however their presence is interesting. It means that there is also an attractive interaction between the dipole and the interface. Its origin may be due to the gradient of the dipole field that has the direction towards the dipole. The well known radiation pressure of a plane wave on a plane interface the force is always negative for any type of interface [10] however unlike our case there are no field gradients. This observation needs an additional study.

As follow from the expressions for the contributions of the force the grows of the force by approaching the dipole to the interface is due to the evanescent components of the dipole field (integration over $k_{1}<k_{\rho}<\infty$ ).

Figure 3 shows the dependence of the total force that the dipole exerted on the interface. As seen, with parameters used in the calculations the maximal force is still too low $\left(10^{-3} \mathrm{fN}\right)$ in order to explain the experimental observations. However, in the calculations of the resulting force we used the value of the bare molecular polarizability without the surface enhancement effects modifying the molecular response and mentioned above as a key element in the experiments [2,3]. In order to include the enhancement in the description we may multiply the maximal force in Figure 3 on an enhancement factor. The reasonable value for this factor is accepted in recent publications is of order of $10^{7}$ [13]-[16]. With this factor in mind we arrive to the theoretical values of several hundreds $\mathrm{fN}$ closed to those claimed in the experiments. Therefore, the model proposed here for the analysis of the radiation forces exerted by the emitting dipole on the interface may be consid- 


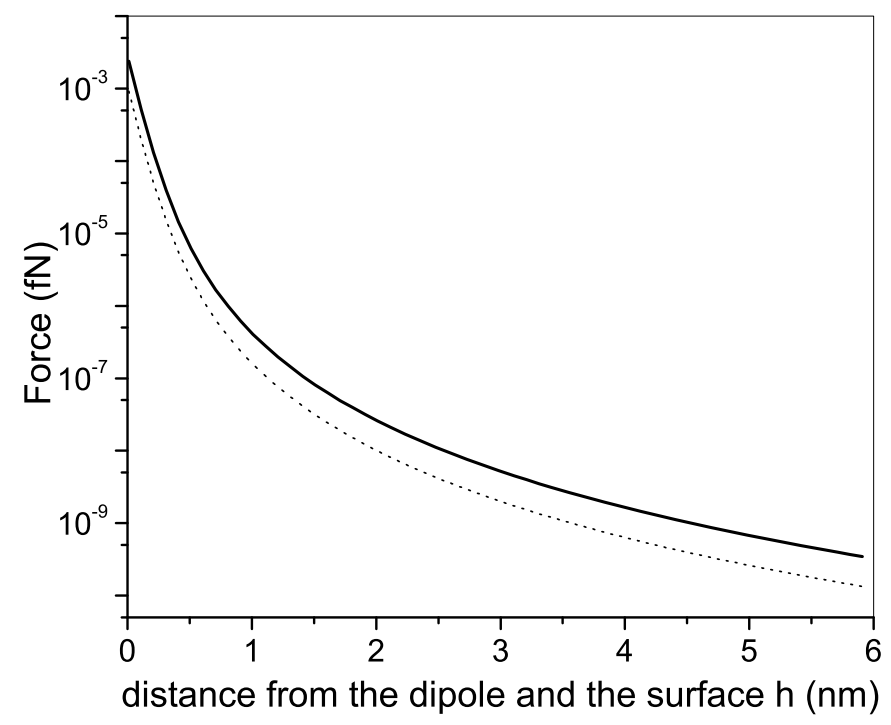

FIG. 3 Total force exerted by a dipole with unit dipole moment exerted on the dielectric (dot line) and metal (solid line) interfaces.

ered as consistent enough in order to be as the starting point for more detailed calculations.

\section{ACKNOWLEDGEMENTS}

I acknowledge support from MIIN FIS2008-00114 (Spain), Fundació Cellex Barcelona and A. V. Chaplik for fruitful discussions.

\section{References}

[1] C. F. Bohren, and D. R. Huffman, Absorption and Scattering of Light by Small Particles (John Wiley it Sons, New York, 1983).

[2] S. Rao, S. Balint, P. Lovhaugen, M. Kreuzer, and D. Petrov, "Measurement of mechanical forces acting on optically trapped dielectric spheres induced by surface-enhanced Raman scattering" Phys. Rev. Lett. 102, 087401 (2009).

[3] A. Zhdanov, S. Rao, A. Fedyanin, and D. Petrov, “Experimental anal- ysis of recoil effects induced by fluorescence photons" Phys. Rev. E 80, 046602 (2009).

[4] L. P. Ghislain, and W. W. Webb, "Scanning force microscope using an optical trap" Opt. Lett. 18, 1678-1680 (1993).

[5] L. P. Ghislain, N. A. Switz, and W. W. Webb, "Measurement of small forces using an optical trap" Rev. Sci. Instrum. 65, 27622768 (1994).

[6] E. C. Le Ru, and P. G. Etchegoin, Principles of Surface-Enhanced Raman Spectroscopy (Elsevier, Oxford, 2009).

[7] D. A. Long, The Raman Effect: A Unified Treatment of the Theory of Raman Scattering by Molecules (John Wiley a Sons, New York, 2002).

[8] L. Novotny, and B. Hecht, Principles of Nano-Optics (Cambridge University Press, Cambridge, 2006).

[9] R. R. Chance, A. Prock, and R. Silbey, "Lifetime of an emitting molecule near a partially reflecting surface" J. Chem. Phys. 60, 2744-2748 (1974).

[10] J. A. Stratton, Electromagnetic Theory (McGraw-Hill Book Company, New York, 1941).

[11] P. W. Milonni, and R. W. Boyd, "Recoil and photon momentum in a dielectric" Laser Phys. 15, 1432-1438 (2005).

[12] D. H. Bradshaw, Z. Shi, and R. W. Milonni, "Electromagnetic momenta and forces in dispersive dielectric media" Opt. Commun. 283, 650-656 (2010).

[13] A. Otto, "On the significance of Shalaev 'hot spots' in ensemble and single-molecule SERS by adsorbates on metallic films at the percolation threshold" J. Raman Spectrosc. 37, 937-947 (2006).

[14] P. J. G. Goulet, and R. F. Aroca, "Distinguishing individual vibrational fingerprints: single-molecule surface-enhanced resonance Raman scattering from one-to-one binary mixtures in LangmuirBlodgett monolayers" Anal. Chem. 79, 2728-2734 (2007).

[15] E. C. L. Ru, E. Blackie, M. Meyer, and P. G. Etchegoin, "Surface enhanced Raman scattering enhancement factors: a comprehensive study" J. Phys. Chem. C 111, 13794-13803 (2007).

[16] P. G. Etchegoin, and E. C. Le Ru, "A perspective of single molecule SERS: current status and future challenges" Phys. Chem. Chem. Phys. 10, 6079-6089 (2008). 